\title{
Glutathione S-transferase Pi expression predicts response to adjuvant chemotherapy for stage $C$ colon cancer: a matched historical control study
}

\author{
Lucy Jankova', Graham Robertson ${ }^{1}$, Charles Chan², King L Tan³, Maija Kohonen-Corish', Caroline L-S Fung ${ }^{3}$, \\ Candice Clarke ${ }^{3}$, Betty P C Lin², Mark Molloy ${ }^{5}$, Pierre H Chapuis ${ }^{6}$, Les Bokey ${ }^{6}$, Owen F Dent ${ }^{6 *}$ and Stephen J Clarke ${ }^{7}$
}

\begin{abstract}
Background: This study examined the association between overall survival and Glutathione S-transferase Pi (GST Pi) expression and genetic polymorphism in stage $\mathrm{C}$ colon cancer patients after resection alone versus resection plus 5-fluourouracil-based adjuvant chemotherapy.

Methods: Patients were drawn from a hospital registry of colorectal cancer resections. Those receiving chemotherapy after it was introduced in 1992 were compared with an age and sex matched control group from the preceding period. GST Pi expression was assessed by immunohistochemistry. Overall survival was analysed by the Kaplan-Meier method and Cox regression.

Results: From an initial 104 patients treated with chemotherapy and 104 matched controls, 26 were excluded because of non-informative immunohistochemistry, leaving 95 in the treated group and 87 controls. Survival did not differ significantly among patients with low GST Pi who did or did not receive chemotherapy and those with high GST Pi who received chemotherapy (lowest pair-wise $p=0.11$ ) whereas patients with high GST Pi who did not receive chemotherapy experienced markedly poorer survival than any of the other three groups (all pair-wise $p<0.01$ ). This result was unaffected by GST Pi genotype.
\end{abstract}

Conclusion: Stage C colon cancer patients with low GST Pi did not benefit from 5-fluourouracil-based adjuvant chemotherapy whereas those with high GST Pi did.

Keywords: Colon cancer, GST Pi, Adjuvant chemotherapy, Survival

\section{Background}

Glutathione S-transferase Pi (GST Pi) is found in the cell nucleus, cytoplasm and mitochondria of a wide range of normal and neoplastic tissues and is expressed in colorectal cancer $[1,2]$. Elevated GST Pi is associated with poor prognosis in many cancers, including colorectal cancer $[3,4]$. Previously we have shown a high level of expression of GST Pi to be associated with adverse histological features and diminished overall survival after adjustment for other prognostic factors in a large series of patients who had a potentially curative resection for stage C colonic adenocarcinoma [5]. That study and

\footnotetext{
* Correspondence: Owen.Dent@netspeed.com.au

${ }^{6}$ Department of Colorectal Surgery, Concord Hospital and Discipline of

Surgery, The University of Sydney, Sydney, NSW 2139, Australia

Full list of author information is available at the end of the article
}

many others in colorectal cancer have been prognostic rather than predictive in that they have examined GST $\mathrm{Pi}$ in consecutive patients who have received the same treatment or mix of treatments, without random allocation to different treatment groups. Predictive biomarkers, on the other hand, can indicate the likely effect of specific adjuvant treatment on patient outcomes such as tumour recurrence or survival.

In colorectal cancer, the prognostic or predictive value of GST Pi expression or genomic polymorphisms for outcomes after chemotherapy has been examined in several studies. However, in most cases, the patients had received palliative treatment for advanced metastatic disease rather than adjuvant treatment in earlier stages and in most reports there was no random allocation to different treatments. Overall, there is some evidence for 
GST Pi as a prognostic biomarker but little evidence for its predictive ability.

As far as we are aware, there has not yet been any attempt to determine whether GST Pi expression or genotype can predict response to adjuvant chemotherapy in stage III patients with colon cancer, yet this is the group of patients for whom adjuvant chemotherapy has most clearly been demonstrated as beneficial in randomised controlled trials [6]. If biomarkers able to predict the results of chemotherapy were found, this could lead to more accurate targeting of treatment to those stage $C$ patients most likely to benefit and to avoidance of inappropriate treatment, with its costs and possible toxicity, in those unlikely gain any advantage.

The ability of a tumour marker to predict response to chemotherapy cannot be determined from a patient series in which some patients were specifically selected for chemotherapy and others were not. At first glance one might simply search for a statistical interaction between the marker and chemotherapy by comparing outcome in four categories of patients: those with a low (or negative) value of the marker who did versus did not receive chemotherapy and those with a high (or positive) value who did versus did not receive chemotherapy. However this would fail to take account of the factors leading to selection or rejection for chemotherapy, which are themselves likely to be associated with survival potential. Comorbidity and advanced tumour stage, for example, are likely to militate against treatment but also to be associated with diminished survival. The ideal research design for identifying the predictive ability of a marker would certainly involve searching for a statistical interaction effect among the four groups defined above, but the patients would need to have been randomly allocated to chemotherapy treatment versus surgery alone [7]. For ethical reasons, because chemotherapy is currently given routinely to a large proportion of stage $\mathrm{C}$ colon cancer patients, it is extremely unlikely that such a randomised trial could now be conducted. An alternative would be to use data from a historical randomised trial with the addition of results for a marker obtained by retrospective histopathological assessment of archived tissue, although this approach is very rare. An example of such a research design is the report by McLornan et al., although that study showed no significant effect from chemotherapy [8].

Using a prospective hospital-based registry of resections for colorectal cancer we were able to construct a historical control group of patients who had a resection for stage $\mathrm{C}$ colonic cancer before chemotherapy was introduced in our hospital in April 1992 and who could be matched with patients from May 1992 to December 2004 who did receive chemotherapy. We acknowledge that this is less than ideal but it is a reasonable surrogate design which can yield legitimate information about the interaction between GST Pi and 5-fluourouracil (5-FU) based chemotherapy in relation to overall survival and it is a feasible design which could be used by others with access to a large historical database and archival tissue bank for colorectal cancer.

The aim of this study was to examine the association between GST Pi expression and overall survival in patients who had received adjuvant chemotherapy after resection of a stage C colon cancer between May 1992 and December 2004 and an age- and sex-matched historical control group of equivalent patients who had been treated by surgery alone before the introduction of adjuvant chemotherapy. A secondary aim was to examine the survival of these patients according to GST Pi genotype.

\section{Results}

Between May 1 1992, when adjuvant chemotherapy was introduced in this hospital, and December 312004 resections for stage $\mathrm{C}$ colon cancer were performed on 263 patients, of whom 104 (40\%) received adjuvant chemotherapy. The proportion receiving chemotherapy increased steadily from $11 \%$ in 1992 to $71 \%$ in 2004 . One hundred and four age- and sex-matched controls were selected from among patients who had a resection for colon cancer between January 1979 and April 1992. Of the total of 208 patients, 26 had insufficient archived tissue for immunohistochemical evaluation, leaving 182; comprising 95 in the chemotherapy group and 87 in the control group. Fifty-two percent in both groups were male and mean ages were 64.1 years (SD 9.5) in the chemotherapy group and 63.6 (SD 10.6) in the control group (Table 1). There was no significant difference between the two groups on any of the 10 pathological characteristics examined in Table 1.

In our earlier detailed study of the prognostic value of GST Pi we showed that the percentage of cells with nuclear staining in the central part of the tumour was the single best measure of GST Pi expression as an independent predictor of overall survival, the optimum cutting point for defining high versus low GST Pi being $\leq$ $40 \%$ versus $>40 \%$ [5]. At this cutting point in the present study high GST Pi occurred in 63 out of 182 patients (35\%). In the chemotherapy group $40 \%$ of patients had high GST Pi as compared with $29 \%$ in the control group, the difference being non-significant $(\mathrm{p}=0.11)$.

The policy of the database is to follow patients yearly for up to 14 completed years. At the time of analysis, 98 patients had died, 3 had been lost to follow-up, 24 had completed 14 years of follow-up and follow-up was continuing for 57. In patients who had not died (including 
Table 1 Background characteristics and tumour pathology in patients who received adjuvant chemotherapy and in age/sex-matched historical controls

\begin{tabular}{lcll}
\hline & $\begin{array}{c}\text { Chemotherapy } \\
\text { Group N=95 }\end{array}$ & $\begin{array}{l}\text { Control } \\
\text { group N=87 }\end{array}$ & Chi $^{\mathbf{2}} \mathbf{p}$ \\
\hline Age (mean, SD) & $64.1(9.5)$ & $63.6(10.6)$ & - \\
Male & $49(52)$ & $45(52)$ & - \\
Tumour site right colon & $50(53)$ & $40(46)$ & 0.37 \\
Size $\geq 5 \mathrm{~cm}$ & $43(45)$ & $43(49)$ & 0.57 \\
Tumour spread beyond & $84(88)$ & $80(92)$ & 0.43 \\
muscularis propria & & & \\
Mucinous or signet ring & $16(17)$ & $10(12)$ & 0.30 \\
adenocarcinoma & & & \\
$\geq 4$ lymph nodes involved & $26(27)$ & $26(30)$ & 0.70 \\
Apical node involved & $11(12)$ & $11(13)$ & 0.83 \\
High grade tumour & $34(36)$ & $31(36)$ & 0.98 \\
Venous invasion present & $20(21)$ & $23(26)$ & 0.39 \\
Free serosal surface involved & $22(23)$ & $23(26)$ & 0.61 \\
Adjacent structure infiltrated & $6(6)$ & $4(5)$ & $0.75^{*}$ \\
\hline
\end{tabular}

*Fisher's exact test.

Number (\%).

those lost) follow-up time ranged from 50 months to 280 months with a median of 126 months.

In the overall pool of 182 patients, survival was significantly poorer in those who had not received chemotherapy than in those who had (hazard ratio [HR] 1.8, 95\% confidence interval [CI] 1.2-2.8, $\mathrm{p}=0.005$ ). It would not be meaningful to draw a similar overall comparison between patients with low and high GST Pi because of the potential for chemotherapy to attenuate the association between GST Pi and survival in the chemotherapy group. However, in patients who had not received chemotherapy, overall survival was significantly poorer among those with high central nuclear GST Pi than among those with low GST Pi (HR 2.3, CI 1.4-3.9, $\mathrm{p}=0.002$ ). Analysis of survival stratified by both high versus low GST Pi and chemotherapy versus no chemotherapy showed no significant difference among patients with low GST Pi who did or did not have chemotherapy and those with high GST Pi who had chemotherapy

Table 2 Five-year overall Kaplan-Meier survival rates for stratified analysis of chemotherapy group versus control group by high versus low nuclear GST Pi expression in central tumour tissue

\begin{tabular}{ll}
\hline & $\begin{array}{l}\text { 5-year survival rate } \\
\text { (95\% confidence interval) }\end{array}$ \\
\hline Low GST Pi with chemotherapy & $68(55-79)$ \\
Low GST Pi without chemotherapy & $61(48-72)$ \\
High GST Pi with chemotherapy & $69(52-82)$ \\
High GST Pi without chemotherapy & 24 (10-42) \\
\hline
\end{tabular}

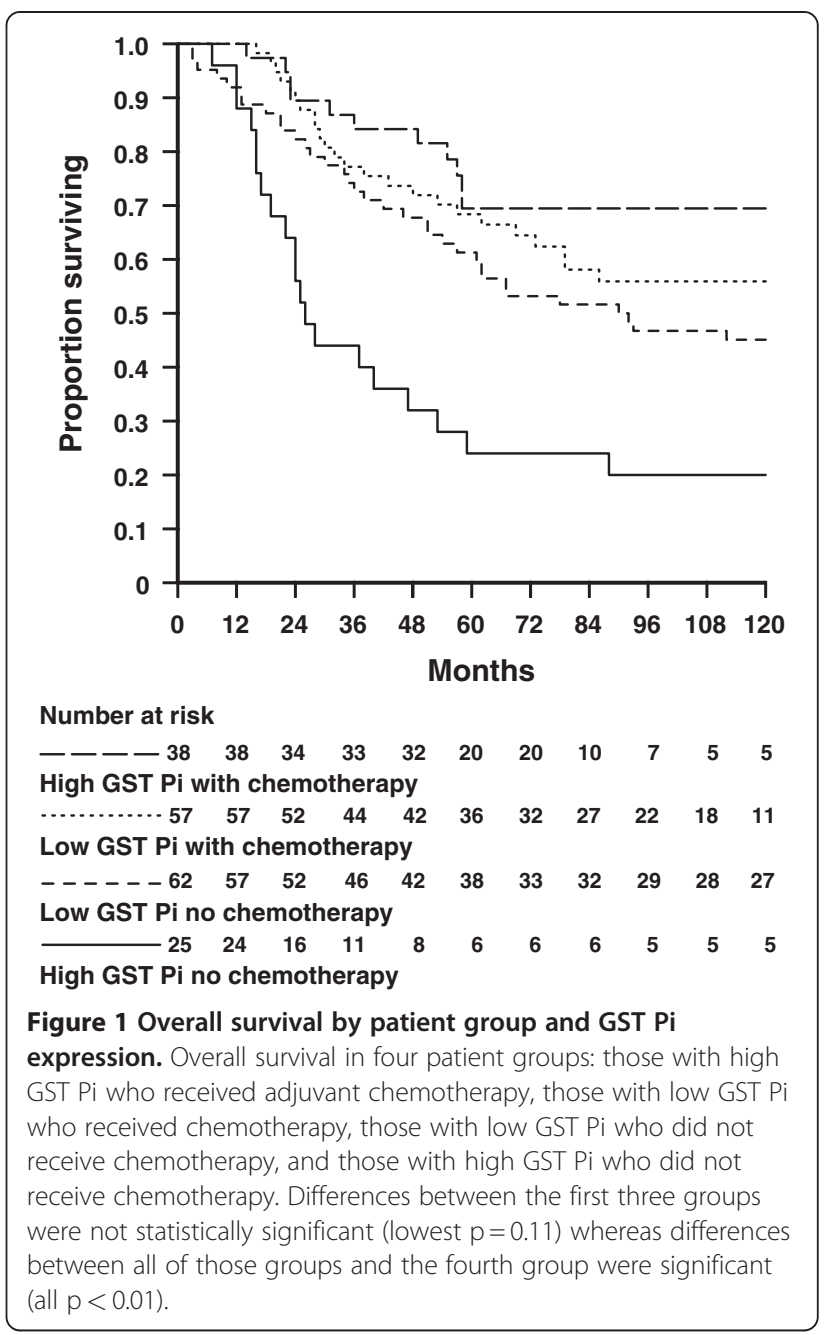

(lowest $\mathrm{p}=0.11$ ) whereas patients with high GST Pi who did not have chemotherapy experienced significantly poorer survival than any of the other groups (all $\mathrm{p}<$ 0.01) (Table 2, Figure 1). This was corroborated by Cox regression modelling where high GST Pi and absence of chemotherapy were coded 2 and the alternatives were coded 1 . The product of these two variables showed a statistically significant interaction $(\mathrm{p}=0.008)$. Furthermore the interaction term remained statistically significant $(\mathrm{p}<0.001)$ when it was included in a model with all variables in Table 1 that had a bivariate $p$ value of $\leq 0.1$ with overall survival (Table 3 ). Thus, while low GST Pi was not associated with any difference in response to chemotherapy, high GST Pi in the absence of chemotherapy predicted a markedly poorer outcome than might otherwise be expected. The implication is that patients with low GST Pi did not benefit from 5FUbased chemotherapy whereas those with high GST Pi clearly did.

The determination of genotype was uninformative in 13 specimens, leaving 169 for analysis; 81 (48\%) having 
Table 3 Overall survival for sex, age, pathology features and GST Pi by chemotherapy interaction

\begin{tabular}{|c|c|c|c|c|}
\hline & $\begin{array}{l}\text { Bivariate } \\
\mathrm{HR}^{*}\end{array}$ & $\begin{array}{l}\text { Bivariate } \\
\mathrm{p} \text { value }\end{array}$ & $\begin{array}{l}\text { Multivariable HR } \\
(95 \% \mathrm{Cl})\end{array}$ & $\begin{array}{l}\text { Multivariable } \\
\mathrm{p} \text { value }\end{array}$ \\
\hline GST Pi by chemotherapy interaction & - & - & $1.4(1.2-1.8)$ & $<0.001$ \\
\hline Male sex & 1.0 & 0.850 & - & - \\
\hline Age $\geq 75$ years & 1.7 & 0.044 & $1.5(0.9-2.6)$ & 0.153 \\
\hline Right colon tumor & 0.7 & 0.063 & $0.8(0.5-1.3)$ & 0.431 \\
\hline Size $\geq 5 \mathrm{~cm}$ & 1.3 & 0.193 & - & - \\
\hline Spread beyond muscularis propria & 3.0 & 0.030 & $1.6(0.6-4.6)$ & 0.356 \\
\hline Mucinous or signet ring & 1.1 & 0.730 & - & - \\
\hline$\geq 4$ lymph nodes involved & 2.5 & $<0.001$ & $1.5(0.97-2.4)$ & 0.071 \\
\hline Apical node involved & 2.8 & $<0.001$ & $2.0(1.1-3.5)$ & 0.021 \\
\hline High grade tumor & 2.3 & $<0.001$ & $1.7(1.1-2.7)$ & 0.016 \\
\hline Venous invasion present & 1.5 & 0.084 & $1.2(0.8-1.9)$ & 0.418 \\
\hline Free serosal surface involved & 3.3 & $<0.001$ & $2.0(1.2-3.1)$ & 0.004 \\
\hline Adjacent structure infiltrated & 1.3 & 0.455 & - & - \\
\hline
\end{tabular}

* Hazard ratio.

the Ile/Ile genotype, 73 (43\%) the Ile/Val genotype and 15 (9\%) the Val/Val genotype, which was similar to other studies [9-11]. The frequency of high GST Pi did not differ significantly by genotype (Ile/Ile $41 \%$, Ile/Val $29 \%$, $\mathrm{Val} / \mathrm{Val} 33 \% ; \mathrm{p}=0.295)$ and there was no difference in the proportions of patients receiving chemotherapy (Ile/ Ile 44\%, Ile/Val 51\%, Val/Val 40\%; $\mathrm{p}=0.637$ ). There was no overall association between genotype and survival (all pair-wise $\mathrm{p}>0.15$ ) and no association between genotype and survival in the chemotherapy group alone (all pairwise $\mathrm{p}>0.42$ ) or in the control group alone (all pairwise $\mathrm{p}>0.13)$. When the joint association between chemotherapy, GST Pi and survival shown in Figure 1 was further stratified by genotype it was again found that, for patients with the Ile/Ile genotype, those with high GST Pi who did not receive chemotherapy had significantly poorer survival than the other three groups (all pair-wise $\mathrm{p}<0.003$ ) and there were no significant differences among those three groups (all pair-wise $\mathrm{p}>$ 0.385 ). The same was true for the Val/Val genotype (the corresponding pair-wise $\mathrm{p}$ values were $\mathrm{p}<0.046$ and $>0.527)$ and there was a non-significant tendency towards the same pattern for the Ile/Val genotype, where there were only nine patients in the high GST Pi/no chemotherapy group. From these results it was concluded that genotype had no influence on the association between survival and the joint effects of chemotherapy and GST Pi expression.

\section{Discussion}

GST Pi is a member of the glutathione-S-transferase superfamily of phase II xenobiotic-metabolizing enzymes that catalyse the conjugation of endogenous and exogenous electrophiles, including reactive oxygen species, toxins, carcinogens and anti-cancer agents, to the nucleophilic thiol group of reduced glutathione (GSH) [12]. A number of studies have concentrated on the connection between aberrant expression of GST isozymes, including GST Pi isozymes, with the development and expression of resistance to chemotherapy drugs as reviewed by Mcllwain et al. [13]. From this perspective an expected result should have been a poor response to chemotherapy in patients with high expression of GST Pi. However our study has shown that, for stage $\mathrm{C}$ colon cancer patients, overall survival was significantly and markedly poorer in patients with high GST Pi who did not receive chemotherapy than in those with high GST $\mathrm{Pi}$ who did. Furthermore, survival in the latter group was no different from that in patients with low GST Pi, whether or not they received chemotherapy. Expressed differently, chemotherapy had no apparent effect on survival in patients with low GST Pi whereas those with high GST Pi appeared to benefit considerably. This finding could be explained by the fact that a major function of GST Pi is not only the phase II metabolism of drugs but to also contribute to the detoxification of endogenously generated reactive oxygen species and the associated potentially toxic macromolecules and lipid peroxides produced in cells. An example of this detoxificant/antioxidant role is the induction of GST Pi as part of the coordinated defence mechanism to protect colonic cells against oxidative injury [14]. The presence of high GST Pi in colon tumours may confer a selective advantage on malignant colon cells, as increased production of reactive oxygen species is a feature of cancer progression [15]. Therefore the poorer overall survival in patients with high GST Pi who did not receive chemotherapy could be attributed to the ability of GST Pi to 
counter the elevated oxidative stress, enhancing persistence of malignant colon cells and thereby leading to poorer survival. This is also consistent with our previous finding of increased GST Pi expression being an independent prognostic factor for reduced overall survival [5]. Furthermore, treatment with diverse chemotherapy drugs tips the redox balance of already stressed cancer cells and elicits selective additional toxicity that ultimately kills them. In this regard the recent reports of the ability of 5-FU to promote oxidative stress in colonic cancer cells $[16,17]$, in addition to its multiple actions as an anti-metabolite, including inhibition of thymidylate synthase, may provide an explanation for the clear benefit of 5-FU based treatment in those patients with high GST Pi (Figure 1). The additional generation of reactive oxygen species by 5 -FU may overwhelm the advantage of high GST Pi in colon cancer cells either by directly increasing the molecular species detoxified by GST Pi enzyme activity and/or by depletion of the available pool of GSH required for conjugation reactions performed by GST enzymes. GSH is also the major anti-oxidant used by cells in non-enzymatic reactions to protect against reactive oxygen species.

Accordingly the implication of our finding is that stage $\mathrm{C}$ colon cancer patients with low intracellular concentrations of GST Pi may not need to be treated with 5FU-based adjuvant chemotherapy whereas those with high GST Pi definitely should be treated. As far as we are aware, this is the first study to demonstrate the predictive value of GST Pi expression in regard to chemotherapy for stage $\mathrm{C}$ colon cancer.

A secondary finding was that GST Pi genotype had no apparent influence on survival or on the association between survival and the joint effects of GST Pi expression and adjuvant chemotherapy. Interest in the effect of GST Pi polymorphisms arose from the concept that variant GST Pi proteins may differentially affect the actions of chemotherapeutic drugs. The single nucleotide polymorphism A313G in exon 5 results in a valine for isoleucine substitution producing a Val/Val variant with reduced GST Pi enzyme activity $[18,19]$. In metastatic colorectal cancer Stoehlmacher et al. found poorer overall survival in patients with the Ile/Ile genotype than in those with the Ile/Val or Val/Val genotypes after combined 5-FU/oxaliplatin chemotherapy $[9,20]$ although this was not replicated by other researchers using the same agents [21]. Also in 5-FU/oxaliplatin-treated patients poorer survival was seen in those with the Ile/ Ile and Ile/Val genotypes compared with the Val/Val genotype [22]. Similarly, significantly poorer survival associated with the Ile/lle genotype as compared with the Ile/Val or Val/Val genotypes was reported in patients treated with FOLFOX-4 [10], although two other studies using FOLFOX showed no difference in survival between genotypes [23,24]. Additionally, a study of oxaliplatin treatment [25] and two others using a mixture of agents and regimens showed no differences between GST Pi genotypes $[11,26]$. All of the above were prognostic studies as all patients had received chemotherapy and there was no random allocation to different treatment groups. A truly predictive study that randomly allocated patients to one of three 5-FU-based treatment strategies also showed no differences between GST Pi genotypes [27] while another study with patients randomised to first-line capecitabine alone versus capecitabine plus irinotecan showed a short, but significant improvement in progression-free survival for the Val/Val genotype only [28]. Two remaining studies included patients with colorectal tumours of all Dukes/TNM stages, not just those with metastatic disease, but produced contradictory results. Holley et al. found no association between GST Pi genotype and overall survival [29] whereas Jones et al. found that the Ile/Ile genotype was associated with decreased survival as compared with the Ile/Val and Val/Val genotypes [30]. Thus the evidence for an association between GST Pi genetic polymorphism and chemotherapeutic treatment and survival (or other patient outcomes) is inconclusive. The reasons may relate to differing study designs; for example retrospective versus prospective, randomized versus non-randomized allocation to treatment; varying stage mixes of patients and hence different mixes of palliative and adjuvant treatment; various chemotherapeutic agents and regimens; and statistical issues such as limited numbers of patients available for analysis. The latter is particularly a problem in relation to the $\mathrm{Val} / \mathrm{Val}$ genotype which has a prevalence of only $8 \%$ to $10 \%$ which may lead to type II errors in small patient samples.

A limitation of the present study is that patients were not randomly allocated to the treatment and control groups. Instead the treatment group comprised all patients selected for chemotherapy since April 1992 when it became available in this hospital, whereas the control patients were drawn from the period from 1979 to April 1992 and individually matched with treated patients on age and sex. However there was no significant difference between the treated group and the control group on 10 other background and tumour pathology characteristics. Comorbidity may have influenced survival and may have differed between the two groups but we were unable to compare them because details of comorbidity were not recorded in the database until 1995. However, as the survival of patients with low GST Pi did not differ significantly between the control and treatment groups, it is most unlikely that differential comorbidity could have accounted for the marked treatment/control survival difference in patients with high GST Pi alone. A further limitation was the relatively 
small number of patients available for analysis; there were 182 in total and 169 for the genotype analyses, which may have limited our ability to find statistically significant differences between some groups. Nevertheless the markedly poorer survival of the high GST Pi/ non-chemotherapy group (Figure 1) suggests that this interaction would be likely to persist in a larger study.

Earlier studies of the association between GST Pi expression and patient outcomes have yielded varying results. A significant independent association between high GST Pi and diminished overall survival was found by Mulder et al. and a similar independent association was found by Sutoh et al. for disease-free survival [3,4]; both studies included colonic and rectal tumours and all four Astler Coller or Dukes stages. Contrary to these results Kim et al. found no association between GST Pi expression and overall survival in patients with stage IV colorectal cancer, all of whom had been treated by 5 FU/oxaliplatin palliative chemotherapy [21]. Given that tumour stage is the most clearly established prognostic variable in colorectal cancer it is reasonable to expect that other prognostic factors, including biomarkers, may behave differently within different stages and also that they may behave differently between the colon and rectum or even at different sub-sites. In our study we focussed on stage $\mathrm{C}$ colonic tumours because of the known beneficial effects of chemotherapy in this patient group and the desire to find biomarkers which would allow more refined targeting of patients most likely to benefit from chemotherapy and to avoid costly and possibly toxic treatment of those who are unlikely to benefit.

\section{Conclusions}

Survival did not differ between patients with low GST $\mathrm{Pi}$, whether or not they had chemotherapy and those with high GST Pi who had chemotherapy, whereas patients with high GST Pi who did not receive chemotherapy had markedly poorer survival than the other three groups. GST Pi genotype was not associated with survival and did not influence the relationship between GST Pi expression, chemotherapy and survival. Given that adjuvant chemotherapy is currently recommended and routinely used in patients with stage $C$ colon cancer it is now unlikely, for ethical reasons, that a randomised trial including a surgery-only control group could be conducted to confirm our findings. However such a study, based on an early randomized trial and archived tissue, may be possible.

\section{Methods}

Information on patients having a resection for colorectal cancer performed by members of the Concord Hospital Department of Colorectal Surgery has been entered into a prospective computer database since 1971 [31,32]. The data set contains information on patient characteristics, co-morbidity, presentation, investigations, surgical management, complications, adjuvant therapy, pathology and follow-up, and has the approval of the South Western Sydney Health Area Ethics Committee. All patients gave written consent personally or through their guardian for pathology specimens and anonymous clinical data to be used for research purposes. From 1981, all resections were performed by specialist colorectal surgeons according to a standardized procedure [33] and acquisition of clinical data has been conducted by a single surgeon ( $\mathrm{P}$. C.). Patients reported here had a resection for clinicopathological stage C colon cancer between 1979 and 2004 inclusive.

\section{Adjuvant chemotherapy}

The chemotherapy regimens utilized involved, for the most part, 6-month courses of bolus injections of 5-FU and folinic acid administered daily for 5 days every 28 days over a total of 6 cycles (Mayo Clinic regimen [34]) or 5-FU and leucovorin repeated weekly for 6 doses with a 2-week rest between (Roswell Park regimen [35]). These regimens were used as they were supported by results from randomized controlled trials on patients with stage $\mathrm{C}$ colon cancer.

\section{Selection of the historical control group}

Patients who received adjuvant chemotherapy were matched individually by sex and age with controls selected from among patients who had a resection for stage C colon cancer between 1979 and April 1992, before radiotherapy and chemotherapy were introduced at this hospital [36]. Matching was by sex because numerous studies have shown sex differences in many epidemiological, clinical and pathological characteristics of colorectal cancer [37] and by age because of the demographic association between advancing age and diminishing survival in the population at large.

\section{Histopathology}

Pathological examination of the resected specimen followed a standard protocol as described previously [38]. Only adenocarcinomas (including mucinous and signet ring cell carcinomas) were included in the data set. Where multiple tumours were present, only the lesion with the most advanced stage was included. Tumour size was measured as the greatest surface dimension. Blocks were taken to demonstrate maximum direct tumour penetration of the bowel wall. Additional blocks were taken specifically to demonstrate the relationship between tumour and any adherent structure or tissue [39] as well as lines of resection and the free serosal surface [40]. Venous invasion by tumour referred to involvement 
of thick or thin walled veins, either within or beyond the bowel wall. When doubt existed as to whether a structure involved was a vein, a negative finding was recorded. Tumour grade was assessed taking into account the degree of differentiation and anaplasia, the nature of the tumour margin (pushing or infiltrating) and the presence and prominence of vascular invasion [31]. An apical lymph node was defined as the most proximal of any nodes found within $1 \mathrm{~cm}$ of the ligation of a named vessel as the apex of a pedicle [41]. All pathological characteristics analyzed were looked for in every specimen and their presence or absence recorded explicitly. There were no missing data on any original database variable. Tumours were staged according to the Australian Clinicopathological Staging System for colorectal cancer which accommodates sub-stages compatible with other clinicopathological staging systems such as Tumour Nodes Metastases [42]. A stage $C$ tumour was defined as one with lymph node metastasis but no systemic metastasis and no tumour present in the proximal, distal or deep lines of resection histologically.

\section{Tissue microarray construction}

Tissue micro arrays (TMA) for the assessment of GST Pi were constructed using an Advanced Tissue Arrayer ATA-100 (Chemicon, Temecula, Ca). $1.0 \mathrm{~mm}$ cores were taken from carefully selected, morphologically representative areas of the original paraffin blocks and arrayed into freshly made recipient paraffin blocks. As it is known that there is heterogeneity within colorectal cancers, we took cores from (a) the central part of the tumour, avoiding the luminal surface, the tumour edge and areas of necrosis, (b) the deep invasive tumour front at the interface between the tumour and non-neoplastic tissue, and also (c) adjacent normal mucosa.

\section{Immunohistochemistry}

GST Pi (1:20, Abcam, ab17088, Cambridge, U.K.) immunohistochemistry was carried out using DAKO Autostainer (DAKO, Glostrup, Denmark). Following dewaxing and rehydration, antigen retrieval was performed in a water bath $\left(95^{\circ} \mathrm{C}\right)$ for 30 minutes using sodium citrate (pH 6.0) Target Retrieval Solution S1699 (DAKO, Glostrup, Denmark). Endogenous peroxidases were blocked with $3 \%$ hydrogen peroxide for 5 minutes. Nonspecific binding sites were blocked with Protein Block (DAKO, Glostrup, Denmark) for 10 minutes. The sections were incubated with diluted GST Pi antibody for 1 hour at room temperature, followed by secondary reagent EnVision + Dual Link System-HRP (DAB+) K4065 (DAKO, Glostrup, Denmark) for 30 minutes. Staining was completed by a 10 minute incubation with 3,3diaminobenzidine $(\mathrm{DAB}+)$ substrate-chromogen. After buffer wash the slides were counterstained with haematoxylin, dehydrated and mounted.

\section{Immunohistochemical evaluation}

Immunoreactivity for GST Pi was assessed independently by three experienced pathologists (K.T., C.F., C.C.) who were unaware of the patients' clinical characteristics, other histopathological data and survival. Tissue cores from the central part of the tumour and the invasive front were assessed separately in each sample, as was the presence of nuclear and cytoplasmic staining in the tumour epithelial cells. The intensity of staining was graded as 0 (no staining), 1 (weak staining), 2 (intermediate staining), 3 (strong staining). The percentage of stained cells (hereinafter termed "percentage stained") was recorded as a quasi-continuous variable coded $0 \%$, $1 \%, 10 \% . .90 \%, 100 \%$. When there were discrepancies between the observers, the slides were reviewed and a consensus reached. To find the optimum dichotomy for percentage stained in relation to survival the distribution of percentage stained was first dichotomized at $0 \%$ versus $1-100 \%$ and survival curves with the associated $p$ value were obtained. The cutting point was then raised in steps of $10 \%(0-9 \%$ vs. $10-100 \%, 0-20 \%$ vs. $30-100 \%$ ... $0-90 \%$ vs. $100 \%)$ and the separation of curves and p value recorded at each step. This process yielded the optimum cutting point giving the greatest separation of survival curves [43].

\section{Genotyping}

Archival paraffin block sections of all the lymph nodes resected from each patient were first reviewed by a pathologist (CC), who selected one normal cancer-free lymph node from each patient for subsequent analysis. A core biopsy was taken from the tissue block and DNA was extracted with the Puregene DNA Isolation Kit (Gentra, Minneapolis, MN) as previously described [44,45]. A custom Taqman SNP Genotyping Assay (Applied Biosystems, Foster City, CA) was used for genotyping. The primer and probe sequences for GSTP1 were as follows:

Forward primer 5'-CCTGGTGGACATGGTGAATG-3'; Reverse primer 5' - TGGTGCAGATGCTCACATAGTTG-3'; Probe 1 (VIC-labelled) 5'-TGCAAATACATCTCC-3'; Probe 2 (FAM-labelled) 5'-CTGCAAATACGTCTCC-3' [27].

The DNA samples were diluted to $\sim 5 \mathrm{ng} / \mathrm{ml}$ and tested in triplicate. Each $10 \mu \mathrm{ml}$ reaction mix contained $5 \mu \mathrm{l}$ of Taqman Universal PCR Master Mix (Applied Biosystems), $2 \mu \mathrm{l}$ of $5 \mathrm{x}$ SNP Genotyping Assay, and $3 \mu \mathrm{l}$ ( $15 \mathrm{ng}$ ) of DNA. The PCR reactions and SNP analysis were carried out on the ABI 7900 (Applied Biosystems), with PCR conditions as follows: $50^{\circ} \mathrm{C}(2 \mathrm{~min}) ; 95^{\circ} \mathrm{C}$ 
(10 min); 40 cycles of $95^{\circ} \mathrm{C}(15 \mathrm{~s})$ followed by $60^{\circ} \mathrm{C}$ (1 min).

\section{Follow-up and survival}

Apart from patients lost to follow-up, all patients were followed annually until death or for up to 14 years or to December 31, 2009. Overall survival time was measured from resection until the date of death due to any cause, the censoring date being the date of last follow-up for those surviving or the date of last contact for those lost to follow-up.

\section{Statistical analysis}

The chi-squared test or Fisher's exact test were used to examine the statistical significance of differences in proportions. Comparisons of survival time between strata of binary variables were made with the Kaplan-Meier method and log-rank test. Proportional hazards regression and the Wald test were used in multivariable modeling with product terms to identify potential interactions. The assumption of proportional hazards was assessed by examining plots of log cumulative hazard for parallelism and in no case was it materially violated in any variable included in a regression model. The level for two-tailed statistical significance was $\mathrm{p} \# 0.05$ with confidence intervals (CI) at the $95 \%$ level. Analyses were performed with SPSS 15.0 for Windows (SPSS Inc., Chicago, Il. USA).

\section{Abbreviations}

GST Pi: Glutathione S-transferase Pi; 5FU: 5-fluourouracil; GSH: The nucleophilic thiol group of reduced glutathione.

\section{Competing interests}

None of the authors have any financial or non-financial competing interests in relation to this paper.

\section{Acknowledgements}

The authors wish to thank all members of the multidisciplinary team managing these patients, namely: G. Stone, nurse unit manager; P. Davey, social worker; E. Galt, clinical psychologist; I. Whitely, stomal therapist; S. Singh, dietician; S. Khatri, cancer care coordinator; G. Sinclair, database manager; Beale, medical oncologist; P. Stewart, M. Rickard, C. Young, A. Keshava, surgeons. Before 2001 Dr R.C. Newland examined and reported on over $90 \%$ of the surgical specimens and reviewed the remainder. N. Currey assisted with the GST Pi genotyping. This work was partly funded by a Cancer Institute NSW Translational Program Grant.

\section{Author details}

${ }^{1}$ Cancer Pharmacology Unit, ANZAC Research Institute, Concord Hospital, The University of Sydney, Sydney, NSW 2139, Australia. ${ }^{2}$ Department of Anatomical Pathology, Concord Hospital and Discipline of Pathology, The University of Sydney, Sydney, NSW 2139, Australia. ${ }^{3}$ Department of Anatomical Pathology, Concord Hospital, Sydney, NSW 2139, Australia. ${ }^{4}$ Cancer Research Program, Garvan Institute of Medical Research and St Vincent's Clinical School, University of New South Wales, Sydney, NSW 2052, Australia. ${ }^{5}$ Australian Proteome Analysis Facility and Department of Chemistry and Biomolecular Sciences, Macquarie University, Sydney, NSW 2109, Australia. ${ }^{6}$ Department of Colorectal Surgery, Concord Hospital and Discipline of Surgery, The University of Sydney, Sydney, NSW 2139, Australia. ${ }^{7}$ Department of Medicine, Concord Hospital and Discipline of Medicine, The University of Sydney, Sydney, NSW 2139, Australia.

\section{Authors' contributions}

$\sqcup J$ had general oversight of the study, conducted immunohistochemistry, and participated in interpretation of results and writing. GR was involved in the conception and design of the study and interpretation of results of the genotyping. CCh, KT, CF, BL were responsible for the immunohistochemical evaluation and contributed to the conception and design of the study and the interpretation of results. $\mathrm{C} \mathrm{Cl}$ conducted the tissue microarray construction and preparation of data for analysis. MK conducted the genotyping and interpretation of results and contributed to drafts of the paper. MM contributed to the conception and design of the study and critical review of the paper. PC and LB were responsible for the surgery and oversight of the clinical database and contributed to the conception and design of the study. OD contributed to the conception and design of the study, conducted the statistical analyses and drafted the paper. SC contributed to the conception and design of the study, oversaw the adjuvant chemotherapy and was involved in writing the paper and general critical review of the work. All authors read and approved the final manuscript.

Received: 24 December 2011 Accepted: 11 May 2012

Published: 28 May 2012

\section{References}

1. Moscow J, Fairchild C, Madden MJ, Ransom DT, Wieand HS, O'Brien EE, Poplack DG, Cossman J, Meyers CE, Cowan KH: Expression of anionic glutathione-S-transferase and P-glycoprotein genes in human tissues and tumours. Cancer Res 1989, 49:1422-1428.

2. Doğru-Abbasoğlu S, Mutlu-Turkoğlu Ü, Türkoğlu S, Erbil Y, Barbaros U, Uysal M, Aykaç-Toker G: Glutathione S-transferase-pi in malignant tissues and plasma of human colorectal and gastric cancers. J Cancer Res Clin Oncol 2002, 128:91-95.

3. Mulder T, Verspaget H, Sier C, Roelofs HMJ, Ganesh S, Griffioen G, Peters WHM: Glutathione S-transferase $\pi$ in colorectal tumours is predictive for overall survival. Cancer Res 1995, 55:2696-2702.

4. Sutoh I, Kohno H, Nakashima Y, Hishikawa Y, Tabara H, Tachibana M, Kubota $\mathrm{H}$, Nagasue $\mathrm{N}$ : Concurrent expressions of metallothionein, glutathione Stransferase- $\pi$, and P-glycoprotein in colorectal cancers. Dis Colon Rectum 2000, 43:221-232.

5. Tan KL, Jankova L, Chan C, Fung CL-S, Clarke C, Lin PC, Robertson G, Molloy M, Chapuis PH, Bokey L, Dent OF, Clarke SJ: Clinicopathological correlates and prognostic significance of Glutathione S-Transferase Pi expression in 468 patients after potentially curative resection of node-positive colon cancer. Histopathology 2011, 59:1057-1070.

6. Gill S, Loprinzi CL, Sargent DJ, Thomé SD, Alberts SR, Haller DG, Benedetti J, Francini G, Shepherd LE, Seitz JF, Labianca R, Chen W, Cha SS, Heldebrant MP, Goldberg RM: Pooled analysis of fluorouracil-based adjuvant therapy for stage II and stage III colon cancer: who benefits and by how much. J Clin Oncol 2004, 10:1797-1806.

7. Lee CK, Lord SJ, Coates AS, Simes RJ: Molecular biomarkers to individualise treatment: assessing the evidence. Med J Aust 2009, 190:631-636.

8. McLornan DP, Barrett HL, Cummins R, McDermott U, McDowell C, Conlon SJ, Coyle VM, Van Schaeybroeck S, Wilson R, Kay EW, Longley DB, Johnston PG: Prognostic significance of TRAIL signalling molecules in Stage II and III colorectal cancer. Clin Cancer Res 2011, 16:3442-3451.

9. Stoehlmacher J, Park DJ, Zhang W, Yang D, Groshen S, Zahedy S, Lenz HJ: A multivariate analysis of genomic polymorphisms: prediction of clinical outcome to 5-FU/oxaliplatin combination chemotherapy in refractory colorectal cancer. Br J Cancer 2004, 91:344-354.

10. Chen Y-C, Tzeng C-H, Chen P-M, Lin J-K, Lin T-C, Chen W-S, Jiang K-K, Wang H-S, Wang W-S: Influence of HSYP1 I105V polymorphism on cumulative neuropathy and outcome of FOLFOX-4 treatment in Asian patients with colorectal carcinoma. Cancer Sci 2010, 101:530-535.

11. Funke S, Timofeeva M, Risch A, Hoffmeister M, Stegmaier C, Seiler CM, Brenner H, Chang-Claude J: Genetic polymorphisms in GST genes and survival of colorectal cancer patients treated with chemotherapy. Pharmacogenomics 2010, 11:33-41.

12. Okamura T, Singh S, Buolamwini J, Haystead T, Friedman H, Bigner D, Ali-Osman F: Tryosine phosphorylation of the human glutathione S-transferase P1 by epidermal growth factor receptor. J Biol Chem 2009, 284:16979-16989. 
13. Mcllwain CC, Townsend DM, Tew KD: Glutathione S-transferase polymorphisms: cancer incidence and therapy. Oncogene 2006, 25:1639-1648

14. Rodriguez-Ramiro I, Ramos S, Bravo L, Goya L, Martin MA: Procyanidin B2 induces Nrf2 translocation and glutathione S-transferase P1 expression via ERKS and p38-MAPK pathways and protect human colonic cells against oxidative stress. Eur J Nutr 2011, : OnlineFirst 31 October 2011.

15. Schumacker PT: Reactive oxygen species in cancer cells: Live by the sword, die by the sword. Cancer Cell 2006, 10:175-176

16. Hwang IT, Chung YM, Kim JJ, Chung JS, Kim BS, Kim HJ, Kim JS, Yoo YD: Drug resistance to 5-FU linked to reactive oxygen species modulator 1 . Biochem Biophys Res Commun 2007, 359:304-310.

17. Santandreu FM, Valle A, Oliver J, Roca P: Resveratrol potentiates the cytotoxic oxidative stress induced by chemotherapy in human colon cancer cells. Cell Physiol Biochem 2011, 28:219-228.

18. Zimniak P, Nanduri B, Pikuła S, Bandorowicz-Pikuła J, Singhal SS, Srivastava SK, Awasthi S, Awasthi YC: Naturally occurring human glutathione Stransferase GSTP1-1 isoforms with isoleucine and valine in position 104 differ in enzymic properties. Eur J Biochem 1994, 224:893-899.

19. Harries LW, Stubbins MJ, Forman D, Howard GCW, Wolf CR: Identification of genetic polymorphisms at the glutathione S-transferase Pi locus and association with susceptibility to bladder, testicular and prostate cancer. Carcinogenesis 1997, 18:641-644.

20. Stoehlmacher J, Park DJ, Zhang W, Groshen S, Tsao-Wei DD, Yu MC, Lenz H-J: Association between GlutThione S-Transferase P1, P1 and M1 genetic polymorphism and survival in patients with metastatic colorectal cancer. JNCl 2002, 94:936-942.

21. Kim S-H, Kwon H-C, Oh SY, Lee DM, Lee S, Lee S-H, Roh M-S, Kim D-C, Park K-I, Choi H-J, Kim H-J: Prognostic value of ERCC1, Thymidylate Synthase and Glutathione S-Transferase $\pi$ for 5-FU/Oxaliplatin chemotherapy in advanced colorectal cancer. Amer J Clin Oncol 2009, 32:38-43.

22. Jun L, Haiping Z, Beibei Y: Genetic polymorphisms of GST Pi related to response to 5-FU-oxaliplatin-based chemotherapy and clinical outcome in advanced colorectal cancer patients. Swiss Med Wkly 2009, 139:274-278.

23. Ruzzo A, Graziano F, Loupakis F, Rulli E, Canestrari E, Santini D, Catalano V, Ficarelli R, Maltese P, Bisonni R, Masi G, Schiavon G, Giordani P, Giustini L, Falcone A, Tonini G, Silva R, Mattioli R, Floriani I, Magnani M: Pharmacogenetic profiling in patients with advanced colorectal cancer treated with first-line FOLFOX-4 chemotherapy. J Clin Oncol 2007, 25:1247-1254

24. Etienne-Grimaldi M-C, Milano G, Maindrault-Gœebel F, Chibaudel B, Formento J-L, Francoual M, Lledo G, André T, Mabro M, Mineur L, Flesch M, Carola E, de Gramont A: Methylenetetrahydrofolate reductase (MTHFR) gene polymorphisms in colorectal cancer patients. Br J Clin Pharmacol 2010, 69:58-66.

25. Kweekel DM, Koopman M, Antonini NF, Van der Straaten T, Nortier JWR, Gelderblom H, Punt CJA, Guchelaar H-J: GST Pi lle105Val polymorphism correlates with progression-free survival in MCRC patients treated with or without irinotecan: a study of the Dutch Colorectal Cancer Group. Br J Cancer 2008, 99:1316-1321.

26. Le Morvan V, Smith D, Laurand A, Brouste V, Bellott R, Soubeyran I, Mathoulin-Pelissier S, Robert J: Determination of ERCC2 Yys751GIn and GSTP1 lle105Val gene polymorphisms in colorectal cancer patients: relationships with treatment outcome. Pharmacogenomics 2007, 8:1693-1703.

27. Braun MS, Richman SD, Thompson L, Quirke P, Daly C, Adlard JW, Elliott F, Barrett JH, Selby P, Meade AM, Stephens RJ, Parmar MKB, Seymour MT: Predictive biomarkers of chemotherapy efficacy in colorectal cancer: Results from the UK MRC FOCUS trial. J Clin Oncol 2008, 26:2690-2698.

28. Kweekel DM, Gelderblom H, Antonini NF, Van der Straaten T, Nortier JWR, Punt CJA, Guchelaar H-J: Glutathione-S-transferase pi (GSTP1) codon 105 polymorphism is not associated with oxaliplatin efficacy of toxicity in advanced colorectal cancer patients. Eur J Cancer 2009, 45:572-578.

29. Holley SL, Rajagopal R, Hoban PR, Deakin M, Fawole AS, Elder JB, Elder J, Smith V, Strange RC, Fryer AA: Polymorphisms in the glutathione Stransferase mu cluster are associated with tumour progression and patient outcome in colorectal cancer. Int J Oncol 2006, 28:231-236.

30. Jones BA, Christensen AR, Wise JP, Yu H: Glutathione S-transferase polymorphisms and survival in African-American and white colorectal cancer patients. Cancer Epidemiol 2009, 33:249-256.
31. Newland RC, Chapuis PH, Pheils MT, Macpherson JG: The relationship of survival to staging and grading of colorectal carcinoma. A prospective study of 503 cases. Cancer 1981, 47(Newland RC):1424-1429.

32. Newland RC, Chapuis PH, Smyth EJ: The prognostic value of substaging in colorectal carcinoma. A prospective study of 1117 cases with standardized pathology. Cancer 1987, 60:852-857.

33. Bokey EL, Chapuis PH, Dent OF, Mander BJ, Bissett IP, Newland RC: Surgical technique and survival in patients having a curative resection for colon cancer. Dis Colon Rectum 2003, 46:860-866.

34. Poon MA, O'Connell MJ, Moertel CG, Wieand HS, Cullinan SA, Everson LK, Crook JE, Mailliard JA, Laurie JA, Tschetter LK: Biochemical modulation of fluorouracil: evidence of significant improvement of survival and quality of life in patients with advanced colorectal carcinoma. J Clin Oncol 1989, 10:1407-1418

35. Petrelli N, Douglass HO, Herrera L, Russell D, Stablein DM, Bruckner HW, Mayer RJ, Schinella R, Green MD, Muggia FM: The modulation of flurourocil with leucovorin in metastatic colorectal cancer; a prospective randomized phase III trial. J Clin Oncol 1989, 10:1419-1426.

36. Chapuis PH, Bokey EL, Clarke S, Beale P, Dent OF: Adjuvant chemotherapy for stage C colonic cancer in a multidisciplinary setting. ANZ J Surg 2009, 79:685-692.

37. Koo JH, Leong WL: Sex differences in epidemiological, clinical and pathological characteristics of colorectal cancer. J Gastroenterol Hepatol 2010, 25:33-42.

38. Davis NC, Newland RC: Terminology and classification of colorectal adenocarcinoma: the Australian Clinico-pathological Staging System. Aust NZ J Surg 1983, 53:211-221.

39. Darakhshan A, Lin BP, Chan C, Chapuis PH, Dent OF, Bokey EL: Correlates and outcomes of tumour adherence in resected colonic and rectal cancers. Ann Surg 2008, 247:650-658.

40. Keshava A, Chapuis PH, Chan C, Lin BPC, Bokey EL, Dent O: The significance of involvement of a free serosal surface for recurrence and survival following resection of clinicopathological stage $B$ and $C$ rectal cancer. Colorectal Dis 2007, 9:609-618.

41. Gabriel WB, Dukes C, Bussey HJR: Lymphatic spread in cancer of the rectum. Br J Surg 1935, 23:395-413.

42. Fielding LP, Arsenault PA, Chapuis PH, Gathright B, Hardcastle JD, Hermanek $P$, Jass JR, Newland RC: Clinicopathological staging for colorectal cancer: An international documentation (IDS) and an international comprehensive anatomical terminology (ICAT). J Gastroenterol Hepatol 1991, 6:325-344

43. Chan C, Jankova L, Fung C, Clarke C, Robertosn G, Chapuis PH, Bokey L, Lin $B P C$, Dent OF, Clarke S: Fascin expression predicts survival after potentially curative resection of node-positive colon cancer. Am J Surg Path 2010, 34:656-666

44. Kohonen-Corish MR, Daniel JJ, Chan C, Lin BP, Kwun SY, Dent OF, Dhillon VS, Trent RJ, Chapuis PH, Bokey EL: Low microsatellite instability is associated with poor prognosis in stage C colon cancer. J Clin Oncol 2005, 23:2318-2324.

45. Kohonen-Corish MR, Sigglekow ND, Susanto J, Chapuis PH, Bokey EL, Dent OF, Chan C, Lin BP, Seng TJ, Laird PW, Young J, Leggett BA, Jass JR, Sutherland RL: Promoter methylation of the mutated in colorectal cancer gene is a frequent early event in colorectal cancer. Oncogene 2007, 26:4435-4441

\section{doi:10.1186/1471-2407-12-196}

Cite this article as: Jankova et al:: Glutathione S-transferase Pi expression predicts response to adjuvant chemotherapy for stage $C$ colon cancer: a matched historical control study. BMC Cancer 2012 12:196. 